\title{
Closed-Form Expression for the Average Age of Information in a Multi-Source M/G/1 Queueing Model
}

\author{
Mohammad Moltafet and Markus Leinonen \\ Centre for Wireless Communications - Radio Technologies \\ University of Oulu, Finland \\ e-mail: \{mohammad.moltafet, markus.leinonen\}@oulu.fi
}

\author{
Marian Codreanu \\ Department of Science and Technology \\ Linköping University, Sweden \\ e-mail: marian.codreanu@liu.se
}

\begin{abstract}
In the context of the next generation wireless networks, freshness of status update packets is essential for enabling the services where a destination needs the most recent measurements of various sensors. In this paper, we study the information freshness of a multi-source M/G/1 first-come firstserved (FCFS) queueing model, where each source independently generates status update packets according to a Poisson process. The information freshness of the status updates of each source is evaluated using the average age of information (AoI). To this end, we derive a closed-form expression for the average AoI of each source. As particular cases of our general expressions, we also derive closed-form expressions of the average AoI for both multi-source $M / M / 1$ and single-source $M / G / 1$ queueing models.

Index Terms- Information freshness, age of information (AoI), multi-source M/G/1 queueing model.
\end{abstract}

\section{INTRODUCTION}

Recently, various services in wireless sensor networks (WSNs) such as Internet of Things and cyber-physical control applications have attracted both academic and industrial attention. In these networks, low power sensors may be deployed to send their status updates about a random process to interested destinations [1]-[3]. The status updates can include different quantities such as temperature of a specific environment (room, greenhouse, etc.), and a vehicular status (position, acceleration, etc.) [1]. In order to have appropriate control commands or decision making in the system, the destination needs the most recent measurements of different sensors. Accordingly, the key enabler for such systems is the freshness of the sensor's information at the destinations.

The traditional metrics such as throughput and delay can not fully characterize the information freshness [2]-[4]. Recently, the age of information (AoI) was proposed as a destination centric metric to measure the information freshness [4]-[6]. The most common metrics of the AoI include average AoI, peak AoI, and effective AoI [2], [7], [8].

From the viewpoint of queueing theory, AoI was first studied in [4] where the authors derived the average AoI for a single-source M/M/1 first-come first-served (FCFS) queueing model. In [8], the authors proposed peak AoI as an alternative metric to evaluate the information freshness. The average AoI and average peak AoI for different packet management policies in an $\mathrm{M} / \mathrm{M} / 1$ queueing model were derived in [9]. The authors of [10] derived the average AoI for a multi-source M/M/1 FCFS queueing model with two sources. The authors of [11] considered a multi-source M/G/1 queueing system and optimized the different arrival rates of each source to minimize the peak AoI. The authors of [12] derived the closed-form expression for the average AoI and average peak AoI in a multi-source M/G/1/1 preemptive queueing model. In [3], the authors introduced a new technique based on stochastic hybrid systems to evaluate the AoI in finite-state continuous-time queueing systems. In other words, this technique can not be used for queueing models with the infinite queue size.

In this work, we derive a closed-form expression for the average AoI of the different sources in a multi-source FCFS M/G/1 queueing model. To the best of our knowledge, such a result has not been derived earlier for this queueing model. Differently from [4], [10]-[14], we consider both multiple sources and an FCFS M/G/1 queueing model which has an infinite queue size. Our result is the most general in the sense that it can be used to derive the average AoI of both the single-source FCFS M/M/1 queueing model in work [4], and the single-source FCFS M/G/1 queueing model in work [14]. Moreover, we derive the average AoI for a multi-source M/M/1 queueing model, which was earlier addressed in work [10]. However, the authors in [10] derived an approximation for the average AoI of the considered queueing model, resulting in a different expression as derived in this paper.

The rest of this paper is organized as follows. The system model and AoI definition is presented in Section II. The closed-form expression for the average AoI for the multisource M/G/1 queueing model is derived in Section III. Evaluation of the AoI is presented in Section IV. Finally, the concluding remarks are expressed in Section V.

\section{System Model And Definitions}

We consider a system consisting of a set of independent sources denoted by $\mathcal{C}=\{1, \ldots, C\}$ and one server. Each source observes a random process, which can represent, e.g., temperature, vehicular speed or location, at random time instants. A remote destination is interested in timely information 


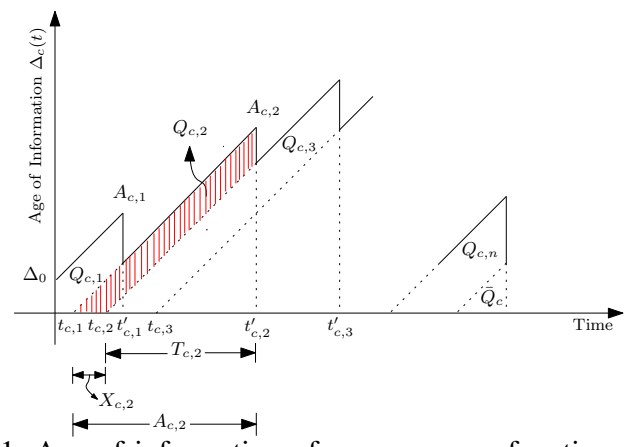

Fig. 1: Age of information of source $c$ as a function of time.

about the status of these random processes. Status updates are transmitted as packets, containing the measured value of the monitored process and a time stamp representing the time when the sample was generated.

For each source, the AoI at the destination is defined as the time elapsed since the last successfully received packet was generated. Next, we give a formal definition of the AoI.

Definition 1 (AoI). Let $t_{c, i}$ denote the time instant at which the $i$ th status update packet of source $c$ was generated, and $t_{c, i}^{\prime}$ denote the time instant at which this packet arrives at the destination. At a time instant $\tau$, the index of the most recently received packet of source $c$ is given by

$$
N_{c}(\tau)=\max \left\{j \mid t_{c, j}^{\prime} \leq \tau\right\},
$$

and the time stamp of the most recently received packet of source $c$ is given by $U_{c}(\tau)=t_{N_{c}(\tau)}$. The AoI of source $c$ at the destination is defined as the random process $\Delta_{c}(t)=t-U_{c}(t)$.

An example of evolution of the AoI with time is shown in Fig. 1. As it can be seen, $\Delta_{c}(t)$ at the destination increases linearly with time, until the reception of a new status update, when the AoI is reset to the AoI of the newly received status update, i.e., the difference of the current time instant and the time stamp of the newly received update. The most common metric for evaluating the AoI of a source at the destination is the average AoI [2], [7], [8], which is considered in this paper. Formally, let $(0, \tau)$ denote an observation interval. Accordingly, the time average AoI of the source $c$ at the destination, denoted as $\Delta_{\tau, c}$, is defined as

$$
\Delta_{\tau, c}=\frac{1}{\tau} \int_{0}^{\tau} \Delta_{c}(t) d t .
$$

The integral in (2) is equal to the area under $\Delta_{c}(t)$ which can be expressed as a sum of disjoint areas determined by a polygon $Q_{c, 1}, N_{c}(\tau)-1$ trapezoids $Q_{c, i}, i=2, \ldots, N_{c}(\tau)$, and a triangle $\bar{Q}_{c}$, as illustrated in Fig. 1. Following the definition of $N_{c}(\tau)$ in (1), $\Delta_{\tau, c}$ can be calculated as follows:

$$
\begin{aligned}
& \Delta_{\tau, c}=\frac{1}{\tau}\left(Q_{c, 1}+\sum_{i=2}^{N_{c}(\tau)} Q_{c, i}+\bar{Q}_{c}\right) \\
& =\frac{Q_{c, 1}+\bar{Q}_{c}}{\tau}+\frac{N_{c}(\tau)-1}{\tau} \frac{1}{N_{c}(\tau)-1} \sum_{i=2}^{N_{c}(\tau)} Q_{c, i} .
\end{aligned}
$$

The average AoI of source $c$, denoted by $\Delta_{c}$, is calculated when $\tau \rightarrow \infty$, i.e., $\Delta_{c}=\lim _{\tau \rightarrow \infty} \Delta_{\tau, c}$. The steady state rate of generating the status update packets of source $c$ is given by $\lambda_{c}=\lim _{\tau \rightarrow \infty} \frac{N_{c}(\tau)}{\tau}$. Then, due to the ergodicity of $Q_{c, i}$ [2], [4], [6]-[16], we have $\Delta_{c}=\lambda_{c} \mathbb{E}\left[Q_{c, i}\right]$.

As depicted in Fig. 1, $Q_{c, i}$ can be calculated by subtracting the area of the isosceles triangle with sides $\left(t_{c, i}^{\prime}-\right.$ $\left.t_{c, i}\right)$ from the area of the isosceles triangle with sides $\left(t_{c, i}^{\prime}-t_{c, i}\right)+\left(t_{c, i}-t_{c, i-1}\right)$. Let the random variable $X_{c, i}=$ $t_{c, i}-t_{c, i-1}$ represent the $i$ th interarrival time of source $c$, i.e., the time elapsed between the generation of packets $c, i-1$ and $c, i$. From here onwards, we refer to the $i$ th packet from source $c$ simply as packet $c, i$. Moreover, let the random variable $T_{c, i}=t_{c, i}^{\prime}-t_{c, i}$ represent the system time of packet $c, i$, i.e., the time interval the packet spends in the system which consists of the sum of the waiting time and the service time. Finally, we have

$$
\begin{aligned}
\Delta_{c} & =\lambda_{c} \mathbb{E}\left[Q_{c, i}\right]=\lambda_{c}\left(\frac{1}{2} \mathbb{E}\left[\left(X_{c, i}+T_{c, i}\right)^{2}\right]-\frac{1}{2} \mathbb{E}\left[X_{c, i}^{2}\right]\right) \\
& =\lambda_{c}\left(\frac{\mathbb{E}\left[X_{c, i}^{2}\right]}{2}+\mathbb{E}\left[X_{c, i} T_{c, i}\right]\right) .
\end{aligned}
$$

\section{AoI in A Multi-Source M/G/1 Queueing Model}

We assume that the packets of source $c$ are generated according to the Poisson process with rate $\lambda_{c}, c \in \mathcal{C}$. Let $\rho_{c}=\lambda_{c} \mathbb{E}[S]$ be the load of source $c$, where $\mathbb{E}[S]$ is the mean service time of each packet in the system. Since packets of each source are generated according to the Poisson process, and the sources are independent, the packet generation in the system follows the Poisson process with rate $\lambda=\sum_{c \in \mathcal{C}} \lambda_{c}$. Moreover, the overall load in the system is given by $\rho=$ $\sum_{c \in \mathcal{C}} \rho_{c}=\mathbb{E}[S] \lambda$. Note that since we do not assume any specific probability distribution function (PDF) for the service time, the considered model is referred to as a multi-source M/G/1 queueing model.

Next, we derive a closed-form expression for the average AoI in (4) for each source in the considered multi-source M/G/1 queueing model. The first term in (4) is easy to compute. Namely, since the interarrival time of source $c$ follows the exponential distribution with parameter $\lambda_{c}$, we have $\mathbb{E}\left[X_{c, i}^{2}\right]=2 / \lambda_{c}^{2}$. However, because the random variables $X_{c, i}$ and $T_{c, i}$ are dependent, the most challenging part in calculating (4) is $\mathbb{E}\left[X_{c, i} T_{c, i}\right]$ which is derived in the following. Let a random variable $S_{c, i}$ denote the service time of packet $c, i$. Since $T_{c, i}=W_{c, i}+S_{c, i}$, we can rewrite term $\mathbb{E}\left[X_{c, i} T_{c, i}\right]$ as

$$
\mathbb{E}\left[X_{c, i} T_{c, i}\right]=\mathbb{E}\left[X_{c, i} W_{c, i}\right]+\mathbb{E}\left[X_{c, i}\right] \mathbb{E}[S],
$$

where $W_{c, i}$ is the waiting time of packet $c, i$ and we used the fact that the interarrival time and service time of the packet $c, i$ are independent. Next, we will derive $\mathbb{E}\left[X_{c, i} W_{c, i}\right]$. In order to calculate $\mathbb{E}\left[X_{c, i} W_{c, i}\right]$, we follow the approach of [10] and characterize the waiting time $W_{c, i}$ by means of two events $E_{c, i}^{\mathrm{B}}$ and $E_{c, i}^{\mathrm{L}}$ as follows:

$$
E_{c, i}^{\mathrm{B}}=\left\{T_{c, i-1} \geq X_{c, i}\right\}, \quad E_{c, i}^{\mathrm{L}}=\left\{T_{c, i-1}<X_{c, i}\right\} .
$$


First, $E_{c, i}^{\mathrm{B}}$ is the event where the interarrival time of packet $c, i$ is brief, i.e., the interarrival time of packet $c, i$ is briefer than the system time of packet $c, i-1$. On the contrary, $E_{c, i}^{\mathrm{L}}$ refers to the complementary event where the interarrival time of packet $c, i$ is long, i.e., the interarrival time of packet $c, i$ is longer than the system time of packet $c, i-1$.

Under the event $E_{c, i}^{\mathrm{B}}$, the waiting time of packet $c, i$ contains two terms: 1) the residual service time to complete serving packet $c, i-1$, and 2) the sum of service times of the packets of other sources $c^{\prime} \in \mathcal{C} \backslash\{c\}$ that must be served before packet $c, i$ according to the FCFS policy. Under the event $E_{c, i}^{\mathrm{L}}$, the waiting time of packet $c, i$ is equal to the sum of service times of the packets of other sources $c^{\prime} \in \mathcal{C} \backslash\{c\}$ that must be served before packet $c, i$ according to the FCFS policy. Thus, the waiting time for packet $c, i$ can be expressed as

$$
W_{c, i}=\left\{\begin{array}{lr}
T_{c, i-1}-X_{c, i}+\sum_{c^{\prime} \in \mathcal{C} \backslash\{c\}} \sum_{j \in \mathcal{M}_{c^{\prime}}^{\mathrm{B}}} S_{c^{\prime}, j}, E_{c, i}^{\mathrm{B}} \\
\sum_{c^{\prime} \in \mathcal{C} \backslash\{c\}} \sum_{j \in \mathcal{M}_{c^{\prime}}^{\mathrm{L}}} S_{c^{\prime}, j}, & E_{c, i}^{\mathrm{L}},
\end{array}\right.
$$

where $\mathcal{M}_{c^{\prime}}^{\mathrm{B}}$ is the set of indices of packets of source $c^{\prime}$ that must be served before packet $c, i$ under the event $E_{c, i}^{\mathrm{B}}$, where $\left|\mathcal{M}_{c^{\prime}}^{\mathrm{B}}\right|=M_{c^{\prime}}^{\mathrm{B}}$, and $\mathcal{M}_{c^{\prime}}^{\mathrm{L}}$ is the set of indices of packets of source $c^{\prime}$ that must be served before packet $c, i$ conditioned on the event $E_{c, i}^{\mathrm{L}}$, where $\left|\mathcal{M}_{c^{\prime}}^{\mathrm{L}}\right|=M_{c^{\prime}}^{\mathrm{L}}, c^{\prime} \in \mathcal{C} \backslash\{c\}$. Fig. 2 depicts the evolution of the AoI as a function of time for the event $E_{c, i}^{\mathrm{B}}$. For the case $E_{c, i}^{\mathrm{B}}$, let us further divide the waiting time $W_{c, i}$ in (7) into two terms $S_{c, i}^{\mathrm{B}_{1}}$ and $S_{c, i}^{\mathrm{B}_{2}}$ as follows. Let

$$
S_{c, i}^{\mathrm{B}_{1}}=T_{c, i-1}-X_{c, i}
$$

represent the residual service time to complete serving packet $c, i-1$ and let

$$
S_{c, i}^{\mathrm{B}_{2}}=\sum_{c^{\prime} \in \mathcal{C} \backslash\{c\}} \sum_{j \in \mathcal{M}_{c^{\prime}}^{\mathrm{B}}} S_{c^{\prime}, j}
$$

represent the sum of service times of the packets of other sources $c^{\prime} \in \mathcal{C} \backslash\{c\}$ that must be served before packet $c, i$. Similarly for the event $E_{c, i}^{\mathrm{L}}$, let

$$
S_{c, i}^{\mathrm{L}}=\sum_{c^{\prime} \in \mathcal{C} \backslash\{c\}} \sum_{j \in \mathcal{M}_{c^{\prime}}^{\mathrm{L}}} S_{c^{\prime}, j}
$$

represent the sum of service times of the packets of the other sources $c^{\prime} \in \mathcal{C} \backslash\{c\}$ that must be served before packet $c, i$. Based on the above definitions in (8), (9), and (10), $\mathbb{E}\left[X_{c, i} W_{c, i}\right]$ in (5) can be calculated as follows:

$$
\begin{aligned}
\mathbb{E}\left[X_{c, i} W_{c, i}\right] & =P\left(E_{c, i}^{\mathrm{B}}\right)\left(\mathbb{E}\left[S_{c, i}^{\mathrm{B}_{1}} X_{c, i} \mid E_{c, i}^{\mathrm{B}}\right]+\right. \\
& \left.\mathbb{E}\left[S_{c, i}^{\mathrm{B}_{2}} X_{c, i} \mid E_{c, i}^{\mathrm{B}}\right]\right)+\mathbb{E}\left[S_{c, i}^{\mathrm{L}} X_{c, i} \mid E_{c, i}^{\mathrm{L}}\right] P\left(E_{c, i}^{\mathrm{L}}\right),
\end{aligned}
$$

where $P\left(E_{c, i}^{\mathrm{B}}\right)$ and $P\left(E_{c, i}^{\mathrm{L}}\right)$ denote the probability of the events $E_{c, i}^{\mathrm{B}}$ and $E_{c, i}^{\mathrm{L}}$, respectively.

Next we derive each term of (11), starting from the expressions for $P\left(E_{c, i}^{\mathrm{B}}\right)$ and $P\left(E_{c, i}^{\mathrm{L}}\right)$. Then, by referring to $\mathbb{E}\left[S_{c, i}^{\mathrm{B}_{1}} X_{c, i} \mid E_{c, i}^{\mathrm{B}}\right], \mathbb{E}\left[S_{c, i}^{\mathrm{B}_{2}} X_{c, i} \mid E_{c, i}^{\mathrm{B}}\right]$, and $\mathbb{E}\left[S_{c, i}^{\mathrm{L}} X_{c, i} \mid E_{c, i}^{\mathrm{L}}\right]$ in (11) as the first, the second, and the third conditional expectation term of (11), we present elaborate derivations of each of them in Sections III-1, III-2, and III-3, respectively. The following lemma gives the expression for $P\left(E_{c, i}^{\mathrm{B}}\right)$ and $P\left(E_{c, i}^{\mathrm{L}}\right)$ in (11).

Lemma 1. For each $c \in \mathcal{C}$, the probabilities of the events $E_{c, i}^{\mathrm{B}}$ and $E_{c, i}^{\mathrm{L}}$ in (6) are calculated as follows:

$$
\begin{gathered}
P\left(E_{c, i}^{\mathrm{B}}\right)=\frac{L_{S}\left(\lambda_{c}\right)\left(\lambda+(\rho-1) \lambda_{c}\right)-\sum_{c^{\prime} \in \mathcal{C} \backslash\{c\}} \lambda_{c^{\prime}}}{\lambda L_{S}\left(\lambda_{c}\right)-\sum_{c^{\prime} \in \mathcal{C} \backslash\{c\}} \lambda_{c^{\prime}}}, \\
P\left(E_{c, i}^{\mathrm{L}}\right)=\frac{(1-\rho) \lambda_{c} L_{S}\left(\lambda_{c}\right)}{\lambda L_{S}\left(\lambda_{c}\right)-\sum_{c^{\prime} \in \mathcal{C} \backslash\{c\}} \lambda_{c^{\prime}}},
\end{gathered}
$$

where $L_{S}\left(\lambda_{c}\right)=\mathbb{E}\left[e^{-\lambda_{c} S}\right]$ is the Laplace transform of the service time at $\lambda_{c}$.

Due to the space limitations, the proof is given in [17]. The relation between the Laplace transform of the system time and the service time is given as follows [18, Sect. 5.1.2]:

$$
L_{T}(a)=\frac{(1-\rho) a L_{S}(a)}{a-\lambda\left(1-L_{S}(a)\right)} \triangleq H_{S}(a),
$$

where we used the notation $H_{S}(a)$ to emphasize that $L_{T}$ is a function of the Laplace transform of the service time.

1) The First Conditional Expectation Term of (11): Let us now focus on the first conditional expectation term $\mathbb{E}\left[S_{c, i}^{\mathrm{B}_{1}} X_{c, i} \mid E_{c, i}^{\mathrm{B}}\right]$ in (11). According to (8), this term is expressed as follows:

$$
\begin{aligned}
& \mathbb{E}\left[S_{c, i}^{\mathrm{B}_{1}} X_{c, i} \mid E_{c, i}^{\mathrm{B}}\right]=\mathbb{E}\left[T_{c, i-1} X_{c, i} \mid E_{c, i}^{\mathrm{B}}\right]-\mathbb{E}\left[X_{c, i}^{2} \mid E_{c, i}^{\mathrm{B}}\right] \\
& =\int_{0}^{\infty} \int_{0}^{\infty} x t f_{X_{c, i}, T_{c, i-1} \mid E_{c, i}^{\mathrm{B}}}(x, t) d x d t-\int_{0}^{\infty} x^{2} f_{X_{c, i} \mid E_{c, i}^{\mathrm{B}}}(x) d x,
\end{aligned}
$$

where $f_{X_{c, i} \mid E_{c, i}^{\mathrm{B}}}(x)$ is the conditional PDF of the interarrival time $X_{c, i}$ given the event $E_{c, i}^{\mathrm{B}}$ and $f_{X_{c, i}, T_{c, i-1} \mid E_{c, i}^{\mathrm{B}}}(x, t)$ is the conditional PDF of the interarrival time $X_{c, i}$ and system time $T_{c, i-1}$ given the event $E_{c, i}^{\mathrm{B}}$. They are given by the following two lemmas (see the proofs in [17]).

Lemma 2. For each $c \in \mathcal{C}$, the conditional PDF $f_{X_{c, i} \mid E_{c, i}^{\mathrm{B}}}(x)$ is given by

$$
f_{X_{c, i} \mid E_{c, i}^{\mathrm{B}}}(x)=\frac{\lambda_{c} e^{-\lambda_{c} x}\left(1-F_{T_{c, i-1}}(x)\right)}{P\left(E_{c, i}^{\mathrm{B}}\right)} .
$$

Lemma 3. For each $c \in \mathcal{C}$, the conditional $P D F$ $f_{X_{c, i}, T_{c, i-1} \mid E_{c, i}^{\mathrm{B}}}(x, t)$ is given by

$$
f_{X_{c, i}, T_{c, i-1} \mid E_{c, i}^{\mathrm{B}}}(x, t)= \begin{cases}0 & x>t \\ \frac{\lambda_{c} e^{-\lambda_{c} x} f_{T_{c, i-1}}(t)}{P\left(E_{c, i}^{\mathrm{B}}\right)} & x \leq t .\end{cases}
$$

Now, having introduced the conditional PDFs in Lemma 2 and Lemma 3, we can compute the conditional expectation $\mathbb{E}\left[S_{c, i}^{\mathrm{B}_{1}} X_{c, i} \mid E_{c, i}^{\mathrm{B}}\right]$ in (15). Based on Lemma 3, the first term in (15) is calculated as (detailed steps can be found in [17]):

$$
\begin{aligned}
& \mathbb{E}\left[T_{c, i-1} X_{c, i} \mid E_{c, i}^{\mathrm{B}}\right]= \\
& \frac{1}{P\left(E_{c, i}^{\mathrm{B}}\right)}\left(-H_{S}^{\prime \prime}\left(\lambda_{c}\right)+\frac{H_{S}^{\prime}\left(\lambda_{c}\right)}{\lambda_{c}}+\frac{\mathbb{E}\left[W_{c}\right]+\mathbb{E}[S]}{\lambda_{c}}\right) .
\end{aligned}
$$




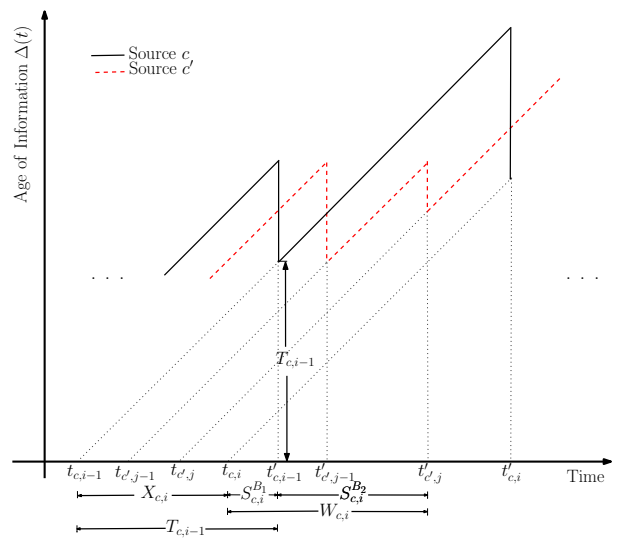

Fig. 2: AoI as a function of time for two sources under brief interarrival times $T_{c, i-1} \geq X_{c, i}$, i.e., under the event $E_{c, i}^{\mathrm{B}}$ in (6).

Using Lemma 2, the second conditional expectation term $\mathbb{E}\left[X_{c, i}^{2} \mid E_{c, i}^{\mathrm{B}}\right]$ in (15) is calculated as follows:

$$
\begin{aligned}
& \mathbb{E}\left[X_{c, i}^{2} \mid E_{c, i}^{\mathrm{B}}\right]= \\
& \frac{1}{P\left(E_{c, i}^{\mathrm{B}}\right)}\left(\frac{2}{\lambda_{c}^{2}}-H_{S}^{\prime \prime}\left(\lambda_{c}\right)+\frac{2 H_{S}^{\prime}\left(\lambda_{c}\right)}{\lambda_{c}}-\frac{2 H_{S}\left(\lambda_{c}\right)}{\lambda_{c}^{2}}\right) .
\end{aligned}
$$

Finally, substituting (18) and (19) in (15), the first conditional expectation $\mathbb{E}\left[S_{c, i}^{\mathrm{B}_{1}} X_{c, i} \mid E_{c, i}^{\mathrm{B}}\right]$ in (11) is given by

$$
\begin{aligned}
& \mathbb{E}\left[S_{c, i}^{\mathrm{B}_{1}} X_{c, i} \mid E_{c, i}^{\mathrm{B}}\right]= \\
& \frac{1}{P\left(E_{c, i}^{\mathrm{B}}\right)}\left(\frac{\mathbb{E}\left[W_{c}\right]+\mathbb{E}[S]}{\lambda_{c}}-\frac{H_{S}^{\prime}\left(\lambda_{c}\right)}{\lambda_{c}}+\frac{2 H_{S}\left(\lambda_{c}\right)}{\lambda_{c}^{2}}-\frac{2}{\lambda_{c}^{2}}\right) .
\end{aligned}
$$

2) The Second Conditional Expectation Term of (11): The conditional expectation $\mathbb{E}\left[S_{c, i}^{\mathrm{B}_{2}} X_{c, i} \mid E_{c, i}^{\mathrm{B}}\right]$ in (11) can be expressed as (detailed steps are presented in [17])

$$
\begin{aligned}
& \mathbb{E}\left[S_{c, i}^{\mathrm{B}_{2}} X_{c, i} \mid E_{c, i}^{\mathrm{B}}\right]=\frac{\mathbb{E}[S] \sum_{c^{\prime} \in \mathcal{C} \backslash\{c\}} \lambda_{c^{\prime}}}{P\left(E_{c, i}^{\mathrm{B}}\right)} \\
& \left(\frac{2}{\lambda_{c}^{2}}-H_{S}^{\prime \prime}\left(\lambda_{c}\right)+\frac{2 H_{S}^{\prime}\left(\lambda_{c}\right)}{\lambda_{c}}-\frac{2 H_{S}\left(\lambda_{c}\right)}{\lambda_{c}^{2}}\right) .
\end{aligned}
$$

3) The Third Conditional Expectation Term of (11): To calculate the third term $\mathbb{E}\left[S_{c, i}^{\mathrm{L}} X_{c, i} \mid E_{c, i}^{\mathrm{L}}\right]$ in (11), we need to determine the quantity $\sum_{c^{\prime} \in \mathcal{C} \backslash\{c\}} M_{c^{\prime}}^{\mathrm{L}}$ which is the total number of packets of other sources $c^{\prime} \in \mathcal{C} \backslash\{c\}$ that must be served before packet $c, i$ under the event $E_{c, i}^{\mathrm{L}}=\left\{T_{c, i-1}<X_{c, i}\right\}$. This is determined by the following lemma which relies on the PASTA (Poisson Arrivals See Time Averages) property of Poisson arrivals [19, Sect. 7.1.2] (see the proof in [17]).

Lemma 4. The total number of packets $\sum_{c^{\prime} \in \mathcal{C} \backslash\{c\}} M_{c^{\prime}}^{\mathrm{L}}$ that must be served before packet c, $i$ under the event $E_{c, i}^{\mathrm{L}}$ is stochastically equal to the number of arrived packets of other sources $c^{\prime} \in \mathcal{C} \backslash\{c\}$ during $T_{c, i-1}$.

Consequently, the third conditional expectation term $\mathbb{E}\left[S_{c, i}^{\mathrm{L}} X_{c, i} \mid E_{c, i}^{\mathrm{L}}\right]$ in (11) can be calculated as (See [17])

$$
\mathbb{E}\left[S_{c, i}^{\mathrm{L}} X_{c, i} \mid E_{c, i}^{\mathrm{L}}\right]=\frac{\sum_{c^{\prime} \in \mathcal{C} \backslash\{c\}} \lambda_{c^{\prime}} \mathbb{E}[S]}{P\left(E_{c, i}^{\mathrm{L}}\right)}\left(H_{S}^{\prime \prime}\left(\lambda_{c}\right)-\frac{H_{S}^{\prime}\left(\lambda_{c}\right)}{\lambda_{c}}\right) .
$$

Finally, substituting the event probabilities $P\left(E_{c, i}^{\mathrm{B}}\right)$ and $P\left(E_{c, i}^{\mathrm{L}}\right)$ in Lemma 1 and the three derived conditional expectation terms (20), (21), and (22) into (11), the average AoI of source $c$ is given as

$$
\begin{aligned}
\Delta_{c} & =\mathbb{E}\left[W_{c}\right]+2 \mathbb{E}[S]+\frac{2 H_{S}\left(\lambda_{c}\right)}{\lambda_{c}}-H_{S}^{\prime}\left(\lambda_{c}\right)-\frac{1}{\lambda_{c}} \\
& +\mathbb{E}[S] \sum_{c^{\prime} \in \mathcal{C} \backslash\{c\}} \lambda_{c^{\prime}}\left(\frac{2}{\lambda_{c}}+H_{S}^{\prime}\left(\lambda_{c}\right)-\frac{2 H_{S}\left(\lambda_{c}\right)}{\lambda_{c}}\right),
\end{aligned}
$$

where the average waiting time for each packet of source $c \in \mathcal{C}, \mathbb{E}\left[W_{c}\right]$, is given as $\mathbb{E}\left[W_{c}\right]=\frac{\mathbb{E}\left[S^{2}\right] \lambda}{2(1-\mathbb{E}[S] \lambda)}[20]$, where $\mathbb{E}\left[S^{2}\right]$ is the second moment of the service time, $H_{S}\left(\lambda_{c}\right)$ is a function of the Laplace transform of the service time presented in (14), and $H_{S}^{\prime}\left(\lambda_{c}\right)$ is the derivative of $H_{S}\left(\lambda_{c}\right)$.

\section{VALIDATION AND RESUlTS}

Owing to the generality of the derived closed-form expression for the average AoI of each source in a multi-source M/G/1 queueing model given in (23), the result can be used to derive AoI expressions for certain particular queueing setups. Accordingly, we derive the average AoI for the multi-source $\mathrm{M} / \mathrm{M} / 1$ and single-source $\mathrm{M} / \mathrm{G} / 1$ queueing models under the FCFS serving policy.

1) Multi-Source FCFS M/M/1 Queueing Model: In the multi-source M/M/1 FCFS queueing model, $C$ independent sources generate packets according to Poisson processes of rates $\lambda_{1}, \lambda_{2}, \ldots, \lambda_{C}$, and the service time follows exponential distribution with parameter $\mu$. Using (23), the average AoI for source $\mathrm{c}$ is given by (detailed steps can be found in [17])

$$
\begin{aligned}
& \Delta_{c}=\frac{1}{\mu}\left(1+\frac{1}{\rho_{c}}\right)+ \\
& \frac{(1-\rho) \rho_{c} \sum_{c^{\prime} \in \mathcal{C} \backslash\{c\}} \rho_{c^{\prime}}}{\mu\left(1-\sum_{c^{\prime} \in \mathcal{C} \backslash\{c\}} \rho_{c^{\prime}}\right)^{2}}\left(\frac{2}{1-\sum_{c^{\prime} \in \mathcal{C} \backslash\{c\}} \rho_{c^{\prime}}}+\frac{1}{\rho_{c}}\right) \\
& +\frac{1}{\mu\left(1-\sum_{c^{\prime} \in \mathcal{C} \backslash\{c\}} \rho_{c^{\prime}}\right)^{2}}\left(\frac{1}{1-\rho}+\frac{2 \sum_{c^{\prime} \in \mathcal{C} \backslash\{c\}} \rho_{c^{\prime}}}{1-\sum_{c^{\prime} \in \mathcal{C} \backslash\{c\}} \rho_{c^{\prime}}}\right) .
\end{aligned}
$$

For case $C=2$, the average AoI for source 1 is given by

$$
\begin{aligned}
& \Delta_{1}=\frac{(1-\rho) \rho_{1} \rho_{2}}{\mu\left(1-\rho_{2}\right)^{2}}\left(\frac{2}{1-\rho_{2}}+\frac{1}{\rho_{1}}\right) \\
& +\frac{\rho_{1}^{2}}{\mu\left(1-\rho_{2}\right)^{2}}\left(\frac{1}{1-\rho}+\frac{2 \rho_{2}}{1-\rho_{2}}\right)+\frac{1}{\mu}\left(1+\frac{1}{\rho_{1}}\right) .
\end{aligned}
$$

Remark 1. It is worth to note that (25) does not coincide with the prior result [10, Eq. (16)]. The deviation of the average AoI of source $1\left(\Delta_{1}\right)$ between the result of [10] and our result in (23) for different arrival rates of source 1 is shown in Fig. 3 where the mean service time is $\mathbb{E}[S]=1$ and the arrival rates of other sources are $\lambda_{c}=0.1, \forall c \in \mathcal{C} \backslash\{1\}$. This deviation is measured as the percentage difference $\frac{\Delta_{1}^{\mathrm{Opt}}-\Delta_{1}^{\mathrm{App}}}{\Delta_{1}^{\mathrm{App}}} \times 100$, where $\Delta_{1}^{\mathrm{Opt}}$ is the average AoI of source 1 using our result in (23) and $\Delta_{1}^{A p p}$ is the average AoI of source 1 using the result of [10]. We 


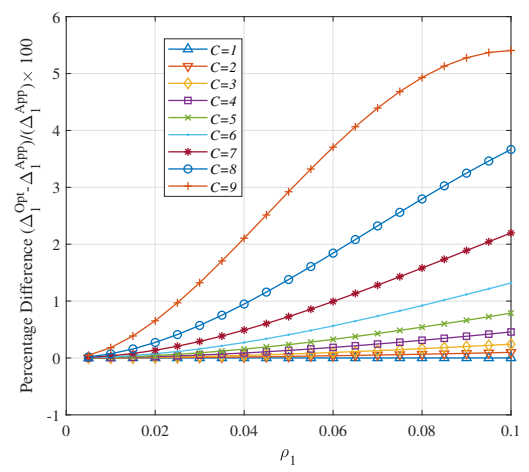

Fig. 3: The deviation between the average AoI of source 1 with using the results of the work [10] and our results with $\mathbb{E}[S]=1$ and $\lambda_{c}=0.1, \forall c \in \mathcal{C} \backslash\{1\}$.

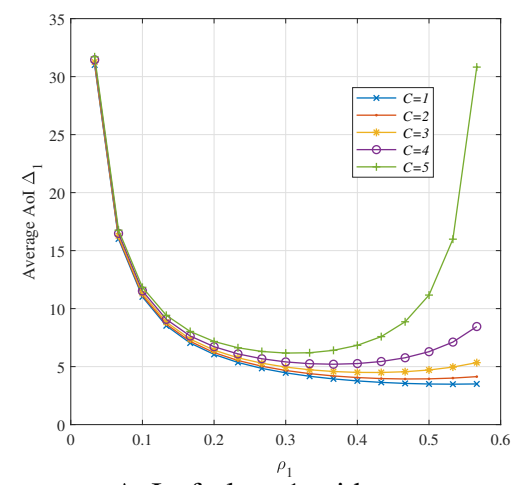

Fig. 4: The average AoI of class 1 with respect to $\rho_{1}$ for different number of classes with $E[S]=1$ and $\lambda_{c}=0.1, \forall c \in \mathcal{C} \backslash\{1\}$.

can observe that the result in [10] provides a lower bound for the average AoI in a multi-source $\mathrm{M} / \mathrm{M} / 1$ queueing model (detailed discussion can be found in [17]).

2) Single-Source FCFS M/G/1 Queueing Model: By setting $C=1$, we have a single-source $\mathrm{M} / \mathrm{G} / 1$ queueing model where interarrival rate of the packets has the exponential distribution with parameter $\lambda$. Then, the average AoI is given by

$$
\Delta=\mathbb{E}[S]+\frac{\lambda \mathbb{E}\left[S^{2}\right]}{2(1-\rho)}+\frac{1-\rho}{\lambda L_{S}(\lambda)} .
$$

It is worth to note that (26) follows the result in [14, Eq. (22)]. The effect of number of classes $C$ on the average AoI of class $1\left(\Delta_{1}\right)$ is shown in Fig. 4. For simplicity, we consider that the service time is exponentially distributed. When $C$ increases, $\Delta_{1}$ increases, as expected. Note that $C$ affects $\Delta_{1}$ non-linearly. As can be seen from the curvatures, when $C$ increases, the optimal value of $\rho_{1}$ decreases.

\section{CONCLUSION}

We considered a multi-source FCFS M/G/1 queueing model and derived the closed-form expression for the average AoI of each source as a function of the Laplace transform of the service time along with certain system parameters. We also showed that the expression for the average AoI for a multisource M/M/1 and a single-source M/G/1 queuing models can be both obtained as particular cases of our general expression.

\section{ACKNOWLEDGEMENT}

M. Codreanu would like to acknowledge the support of the European Union's Horizon 2020 research and innovation programme under the Marie Skłodowska-Curie Grant Agreement No. 793402 (COMPRESS NETS). This research has also been financially supported by Infotech Oulu and Academy of Finland 6Genesis Flagship (grant 318927) and Finnish Foundation for Technology Promotion.

\section{REFERENCES}

[1] P. Papadimitratos, A. D. L. Fortelle, K. Evenssen, R. Brignolo, and S. Cosenza, "Vehicular communication systems: Enabling technologies, applications, and future outlook on intelligent transportation," IEEE Commun. Mag., vol. 47, no. 11, pp. 84-95, Nov. 2009.

[2] A. Kosta, N. Pappas, and V. Angelakis, "Age of information: A new concept, metric, and tool," Foun. and Trends in Net., vol. 12, no. 3, pp. 162-259, 2017.

[3] R. D. Yates and S. K. Kaul, "The age of information: Real-time status updating by multiple sources," IEEE Trans. Inform. Theory, vol. 65, no. 3, pp. 1807-1827, Mar. 2019.

[4] S. Kaul, R. Yates, and M. Gruteser, "Real-time status: How often should one update?" in Proc. IEEE Int. Conf. on Computer. Commun. (INFOCOM), Orlando, FL, USA, Mar. 25-30, 2012, pp. 2731-2735.

[5] S. K. Kaul, R. D. Yates, and M. Gruteser, "Status updates through queues," in Proc. Conf. Inform. Sciences Syst. (CISS), Princeton, NJ, USA, Mar. 21-23, 2012, pp. 1-6.

[6] S. Kaul, M. Gruteser, V. Rai, and J. Kenney, "Minimizing age of information in vehicular networks," in Proc. Commun. Society. Conf. on Sensor, Mesh and Ad Hoc Commun. and Net., Salt Lake City, UT, USA, Jun. 27-30, 2011, pp. 350-358.

[7] A. M. Bedewy, Y. Sun, and N. B. Shroff, "Age-optimal information updates in multihop networks," in Proc. IEEE Int. Symp. Inform. Theory, Aachen, Germany, Jun. 25-30, 2017, pp. 576-580.

[8] M. Costa, M. Codreanu, and A. Ephremides, "Age of information with packet management," in Proc. IEEE Int. Symp. Inform. Theory, Honolulu, HI, USA, Jun. 20-23, 2014, pp. 1583-1587.

[9] — "On the age of information in status update systems with packet management," IEEE Trans. Inform. Theory, vol. 62, no. 4, pp. 1897 1910, Apr. 2016.

[10] R. D. Yates and S. Kaul, "Real-time status updating: Multiple sources," in Proc. IEEE Int. Symp. Inform. Theory, Cambridge, MA, USA, Jul. 1-6, 2012, pp. 2666-2670.

[11] L. Huang and E. Modiano, "Optimizing age-of-information in a multiclass queueing system,” in Proc. IEEE Int. Symp. Inform. Theory, Hong Kong, China, Jun. 14-19, 2015, pp. 1681-1685.

[12] E. Najm and E. Telatar, "Status updates in a multi-stream M/G/1/1 preemptive queue," in Proc. IEEE Int. Conf. on Computer. Commun. (INFOCOM), Honolulu, HI, USA, Apr. 15-19, 2018, pp. 124-129.

[13] E. Najm, R. Yates, and E. Soljanin, "Status updates through M/G/1/1 queues with HARQ," in Proc. IEEE Int. Symp. Inform. Theory, Aachen, Germany, Jun. 25-30, 2017, pp. 131-135.

[14] Y. Inoue, H. Masuyama, T. Takine, and T. Tanaka, "The stationary distribution of the age of information in FCFS single-server queues," in Proc. IEEE Int. Symp. Inform. Theory, Aachen, Germany, Jun. 2530, 2017, pp. 571-575.

[15] A. Kosta, N. Pappas, A. Ephremides, and V. Angelakis, "Age of information and throughput in a shared access network with heterogeneous traffic," in Proc. IEEE Global Telecommun. Conf., Abu Dhabi, United Arab Emirates, Dec. 9-13, 2018, pp. 1-6.

[16] R. Talak, S. Karaman, and E. Modiano, "Minimizing age-of-information in multi-hop wireless networks," in Proc. Annual Allerton Conf. Commun., Contr., Computing, IL, USA, Oct. 3-6, 2017, pp. 486-493.

[17] M. Moltafet, M. Leinonen, and M. Codreanu, "Closed-form expression for the average age of information in a multi-source $M / G / 1$ queueing model," available: http://www.ee.oulu.fi/ zetite/Files/ITW-2019.pdf.

[18] J. N. Daigle, Queueing Theory with Applications to Packet Telecommunication. New York: Springer Science, 2005.

[19] R. Nelson, Probability, Stochastic Processes, and Queueing Theory: The Mathematics of Computer Performance Modeling. New York: Springer Science, 1995.

[20] D. P. Bertsekas, R. G. Gallager, and P. Humblet, Data Networks. Englewood Cliffs, New Jersey: Prentice-Hall International, 1992. 